\title{
Use of Lead (II) Sulfide Nanoparticles as Stabilizer for PMMA Exposed to Gamma Irradiation
}

\author{
Olga Pinheiro Garcia ${ }^{a}$, Marília Cordeiro Carneiro de Albuquerque ${ }^{a}$, Kátia Aparecida da Silva Aquino ${ }^{a *}$, \\ Patricia Lopes Barros de Araujo ${ }^{b}$, Elmo Silvano de Araujo ${ }^{a}$ \\ ${ }^{a}$ Departmento de Energia Nuclear, Universidade Federal de Pernambuco - UFPE, \\ Av. Prof. Luiz Freire, 1000, Cidade Universitária, CEP 50740-540, Recife, PE, Brazil \\ ${ }^{b}$ Universidade Federal Rural de Pernambuco - UFRPE, Av. Dom Manoel de Medeiros, s/n, \\ CEP 50171-000, Recife, PE, Brazil
}

Received: September 17, 2014; Revised: March 25, 2015

\begin{abstract}
Lead (II) sulfide $(\mathrm{PbS})$ were synthesized by sonochemical method and crystals with cubic structure exhibit aggregated nanoparticles with size in the range of 50-100 nm. Commercial Poly(methyl methacrylate) (PMMA) containing the $\mathrm{PbS}$ nanoparticles ( $\mathrm{PbS}-\mathrm{NP}$ ) exposed to gamma irradiation were investigated and both the viscosity-average molar mass $\left(\mathrm{M}_{\mathrm{v}}\right)$ and degradation index (DI) values were measured. Ours results showed decreases in molar mass when the systems were gamma irradiated, i. e., random scission effects that take place in the main chain. On the other hand, DI results showed that the addition of PbS-NP at $0.3 \mathrm{wt} \%$ into the PMMA matrix decreased $100 \%$ the number of main chain scissions. Results about the free radical scavenger action of the PbS-NP were obtained by use of 2,2-diphenyl-1-(2,4,6-trinitrophenyl)-hydrazyl radical (DPPH) and are discussed in this study. Analysis of infrared spectra, refraction index, mechanical, and thermal properties showed influence of the PbS-NP in the physical behavior of PMMA.
\end{abstract}

Keywords: PMMA, nanoparticles, sonochemical, radiation resistance

\section{Introduction}

In recent years, there has been considerable interest in nanoscale chalcogenides due to their remarkable properties and promising application prospects ${ }^{1}$. Among these materials, lead (II) sulfide $(\mathrm{PbS})$ has attracted much attention for its well-known narrow band gap semiconducting features. $\mathrm{PbS}$ is chosen for this work because it is commercially available and can also be synthesized easily. A variety of chemical and physical methods were developed to prepare $\mathrm{PbS}$. Among these methods are: gas-phase synthesis ${ }^{2}$, from the melt, by Bridgman method or gamma irradiation ${ }^{3,4}$, micelles or monolayer surfaces ${ }^{5,6}$, and in aqueous solvents ${ }^{7}$. In addition, template-mediated growth techniques have also been reported to synthesize sulfides nanoparticles in the presence of polymer materials ${ }^{8}$. However, most of the methods have limitation in practice, especially when using noxious compounds, such as $\mathrm{H}_{2} \mathrm{~S}$.

Currently, sonochemical processing has been proven to be an useful technique for generating novel materials with unusual properties. Sonochemical reactions arise from acoustic cavitation phenomenon: the formation, growth, and implosive collapse of bubbles in a liquid medium. This collapse generates a localized hotspot, which has temperatures of about $5000{ }^{\circ} \mathrm{C}$, and very high pressures ${ }^{9}$. A plethora of materials have been synthesized using such extreme conditions, some of them exhibiting improved characteristics ${ }^{10}$.

*e-mail: aquino@ufpe.br
Inorganic-polymer materials have attracted considerable attention in recent years due to their new physical and chemical properties. This material can have specialized properties that cannot be found in their respective single phase and enhanced electrical, mechanical and optical properties have been reported. For example, the $\mathrm{PbS}$ nanoparticles can be embedded in polymer matrix to form high refractive index nanocomposite ${ }^{11}$. In this study, the refractive index of $\mathrm{PbS}$ gelation system increases from 1.5 to 2.5 with increasing $\mathrm{PbS}$ ratio.

On the other hand, radiation resistance of polymeric materials attracts significant interest due to the numerous applications of polymers when radiation exposure is a concern. Radiation resistance of polymers depends on the extent of molecular changes due to the irradiation. In particular, Poly (methyl methacrylate) (PMMA) is commonly used to determine the effects of various additives on polymer radiation stability. The PMMA is produced and formed in rigid bodies showing excellent transparency, good mechanical properties and high resistance to weathering ${ }^{12}$. This polymer is also used in manufacturing of radio-sterilizable medical supplies ${ }^{13}$ or in dosimeters for intense radiation fields ${ }^{14}$, and the behavior of this polymer under radiation has been extensively studied $^{12,14}$, including ours previous studies ${ }^{15}$. PMMA is known to undergo main chain scission when exposed to ionizing radiation. In general, polymer radicals are responsible for changes in physical properties of $\mathrm{PMMA}^{16}$. In particular, gamma irradiation of PMMA, causes main chain scission and hydrogen abstraction from an alpha-methyl or methylene 
group $^{17}$. The evolution of products including monomer, carbon dioxide, carbon monoxide, methane, propane, and hydrogen serve as indicators of polymer degradation ${ }^{12,13,14}$.

In the present investigation, we report the synthesis of $\mathrm{PbS}$ nanoparticles (PbS-NP) by sonochemical route from solution containing lead (II) acetate as metal source and thioacetamide as sulfur source. Films of PMMA/PbS-NP were exposed to gamma irradiation and the effects of inorganic nanoparticles on the viscosity average molar mass $\left(M_{v}\right)$ of gamma irradiated PMMA were studied. Modifications in PMMA structure upon the addition of PbS-NP were analyzed by infrared spectrometry. In addition, free radical scavenging action of $\mathrm{PbS}-\mathrm{NP}$, optical, mechanical and thermal properties of PMMA/PbS-NP films were also discussed. Our findings suggest that $\mathrm{PbS}-\mathrm{NP}$ is suitable additive for improving the properties of PMMA with radiation resistance applications.

\section{Experimental}

\subsection{Synthesis and characterization of $P b S-N P$}

All the reagents used in our experiments were of analytical grade. Lead (II) acetate $\left(\mathrm{Pb}\left(\mathrm{CH}_{3} \mathrm{COO}\right)_{2}\right)$ and thioacetamide $\left(\mathrm{CH}_{3} \mathrm{CSNH}_{2}\right)\left(\mathrm{VETEC}^{\circledR}\right.$, Brazil), absolute ethanol and acetone (DINAMICA ${ }^{\circledR}$, Brazil) were used without further purification. Methyl-ethyl-ketone (MEK) was dried with $\mathrm{Na}_{2} \mathrm{SO}_{4}$ and purified by distillation to prior use.

In a typical procedure, $0.45 \mathrm{~g}$ of $\mathrm{Pb}\left(\mathrm{CH}_{3} \mathrm{COO}\right)_{2}$ and $0.8 \mathrm{~g}$ of $\mathrm{CH}_{3} \mathrm{CSNH}_{2}$ were dissolved in $50 \mathrm{~mL}$ of absolute ethanol in a $100 \mathrm{~mL}$ becker. Then the mixture solution was exposed to ultrasound irradiation in air for $0.5 \mathrm{~h}$ with a high-intensity ultrasonic probe (Sonic, $20 \mathrm{kHz}, 500 \mathrm{~W}$ ) immersed directly into the reaction solution. After this time, the sample was cooled down at room temperature and the black precipitate of $\mathrm{PbS}-\mathrm{NP}$ was separated by centrifugation, washed successively with absolute ethanol, distilled water, acetone, and then dried in a desiccator at room temperature for $24 \mathrm{~h}$. The final product was characterized by Scanning Electron Microscopy (SEM, JEOL JSM- 5900) and X-ray powder diffraction (DRX) on a Siemens D5000 Diffractometer equipped with graphite monochromatic $\mathrm{CuK} \alpha$ radiation $(\lambda=1.5418 \AA$ ) $)$ using a scanning rate of $0.02 \mathrm{deg} / \mathrm{s}$ in the $2 \theta$ range from $10^{\circ}$ to $70^{\circ}$.

\subsection{Preparation of $P M M A / P b S-N P$ films}

Commercial PMMA (Resarbrás, Brazil) was used to prepare PMMA-control and PMMA/PbS-NP films by solventcasting from MEK solvent and slow evaporation in air at room temperature $\left(\approx 27{ }^{\circ} \mathrm{C}\right)$. According to manufacturer information the PMMA has $0.1 \mathrm{wt} \%$ of processing additives and was obtained by free radical polymerization (suspension). The concentrations of PbS-NP used in this study were 0.15; $0.30 ; 0.45$ and $0.60 \mathrm{wt} \%$.

\subsection{Viscosity measurements}

The viscosity measurements of PMMA-control and PMMA/PbS-NP films were carried out in MEK solution at $25.0 \pm 0.1^{\circ} \mathrm{C}$ using an Ostwald viscometer in a thermostatic bath. The intrinsic viscosity of the samples was calculated from the relative viscosity, $\eta_{\text {rel }} \approx v / v_{0} \approx t / t_{0}$, within range of
$1.1-1.9$, where $v$ and $v_{0}$ are the cinematic viscosities of polymer solution and solvent, respectively. The $t$ and $t_{0}$ are flow times of solution and solvent, respectively. Therefore, $\eta_{\text {rel }}$ was calculated from $t / t_{0}$ ratio. The specific viscosity $\left(\eta_{\mathrm{sp}}=\eta_{\text {rel }}-1\right)$ and the reduced viscosity $\left(\eta_{\text {red }}=\eta_{\mathrm{sp}} / \mathrm{C}\right)$, where $\mathrm{C}$ is the concentration of the solution $(0.6 \mathrm{~g} / \mathrm{dL})$, were calculated as well. The intrinsic viscosity [ $\eta]$ was determined by the Solomon-Ciuta equation ${ }^{18}$ :

$[\eta]=\left(\frac{1}{\mathrm{C}}\right) \sqrt{2\left(\eta_{s p}-\ln \eta_{r e l}\right)}$

The viscosity average molar mass $\left(\mathrm{M}_{\mathrm{v}}\right)$ was calculated from the corresponding [ $\eta]$ values through the Mark-Houwink equation ${ }^{19}$ using Equation 1.

$[\eta]=K \mathrm{M}_{\mathrm{v}}^{a}$

where $K$ and $a$ are $6.8 \times 10^{-5} \mathrm{dL} / \mathrm{g}$ and 0.72 , respectively for the MEK-PMMA system at $25^{\circ} \mathrm{C}^{20}$.

Radio-stabilizing action of PbS-NP on PMMA matrix could be assessed by comparison of degradation index parameter $(\mathrm{DI}), \mathrm{DI}=\left(\mathrm{M}_{\mathrm{vo}} / \mathrm{M}_{\mathrm{v}}\right)-1$, for a determined irradiation dose. $M_{v 0}$ and $M_{v}$ are the viscosity average molar mass before and after the gamma irradiation, respectively. DI is obtained from viscosity analysis and reflects the number of main chain scissions per original molecule after irradiation. The protection $(\%)$ of $\mathrm{PbS}-\mathrm{NP}$ in the polymer matrix was obtained by DI values (Protection $(\%)=100 \times\left[\mathrm{DI}_{\mathrm{PMMA}}\right.$ $\left.\left.-\mathrm{DI}_{\mathrm{PMMA} / \mathrm{PbS}-\mathrm{NP}} / \mathrm{DI}_{\mathrm{PMMA}}\right]\right)$.

\subsection{Irradiation of samples}

PMMA-control and PMMA/PbS-NP films were exposed to gamma radiation from a ${ }^{60} \mathrm{Co}$ source (dose rate of $6.13 \mathrm{kGy} / \mathrm{h}$ ) at dose of $25 \mathrm{kGy}$ in air at room temperature for radiation resistance study.

\subsection{Radical scavenging action of PbS-NP}

The 2,2-diphenyl-1-(2,4,6-trinitrophenyl)-hydrazyl radical (DPPH) solution $(0.0024 \mathrm{~g}$ of the DPPH in the $100 \mathrm{~mL}$ ethanol) was mixed with appropriate amount of PbS-NP under vigorous stirring at room temperature for $30 \mathrm{~min}$. The absorbance at $515 \mathrm{~nm}$ was measured against a blank of pure ethanol after the reaction in a UV-vis spectrophotometer (Spectro 22, 108-D and $60 \mathrm{~Hz}$ ). Radical DPPH scavenging capacity $(\% \mathrm{SC})$ of $\mathrm{PbS}-\mathrm{NP}$ was estimated by subtracting blank absorbance, according to Equation 3.

$\% \mathrm{SC}=\left(\mathrm{A}_{\mathrm{s}}-\mathrm{A}_{\mathrm{sn}}\right) \times 100$

Where $\mathrm{A}_{\mathrm{s}}=\mathrm{DPPH}$ ethanol solution absorbance and $\mathrm{A}_{\mathrm{sn}}=\mathrm{DPPH}$ solution absorbance for system with PbS-NP. All tests were conducted in triplicate. The values obtained from $\mathrm{PbS}-\mathrm{NP}$ were compared with the Butylated hydroxytoluene (BHT) (control standard).

\subsection{FT-IR characterization}

The infrared spectra of PMMA-control and PMMA/ PbS-NP films were obtained by Fourier Transform Infrared Spectroscopy (FT-IR) with Bruker-IFS66 equipment in transmittance mode, $4000-400 \mathrm{~cm}^{-1}$ wavenumber region, 75 scans with $4 \mathrm{~cm}^{-1}$ resolution, on $\mathrm{KBr}$ pellets. 


\subsection{Optical, mechanical and thermal properties}

The refractive indexes (RI) of the film with thickness $0.12 \pm 0.01 \mathrm{~mm}$ were measured with an Abbe's refractometer at $25^{\circ} \mathrm{C}$ with 1-Bromonaphtalene as solvent. The measurements were carried out for four samples.

The tensile properties of the films were determined according to ASTM D-882 using an Instron machine EMIC, DL-500 N. The crosshead speed was $10 \mathrm{~mm} / \mathrm{min}$. The tests were carried out at room temperature and the results shown in this study are an average of four samples.

Thermogravimetric analyses were performed in a TGA50 Schimadzu Thermoanalyzer, heating rate $10{ }^{\circ} \mathrm{C} / \mathrm{min}$ in nitrogen atmosphere $(10 \mathrm{~mL} / \mathrm{min})$. DTG results were obtained by taking the derivative of the ratio of the sample weight, $\mathrm{W}$, in a given temperature to the initial weight, $\mathrm{W}_{\mathrm{o}}$, taken with respect time, $\mathrm{d}\left(\mathrm{W} / \mathrm{W}_{\mathrm{o}}\right) / \mathrm{dt}$.

\section{Results and Discussion}

\subsection{PbS-NP characterization}

Among the advantages of the preparation of sulfide particles by sonochemical method are: uniform distribution dispersion of the nanoparticles, a marginally higher surface area, better thermal stability, and phase purity ${ }^{6}$. PbS-NP were obtained by the sonochemical method proposed in this study. The synthesis was carried out within $30 \mathrm{~min}$ of the sonication in ethanol, at room temperature. Two main effects in PbS-NP synthesis can be attributed to ultrasound irradiation. The first effect is favoring dissolution of thioacetamide and formation of sulfide ion, thus accelerating the reaction, and the second is preventing the aggregation of the nanoparticles. During the sonication, bubble collapsing in the liquid medium resulted in an enormous concentration of energy from the conversion of kinetic energy of the liquid motion into heating of the bubble content. The high local temperature and pressure provide favorable conditions for driving the decomposition of the $\mathrm{Pb}$ /thioacetamide complex, giving rise to the formation of sulfides ${ }^{21}$. This fast and simple method allowed the production of PbS-NP as can be seen in DRX and SEM results.

The Figures 1 show the DRX patterns and all peaks can be indexed as cubic phase with obvious (111), (220) and (311) crystal planes (JCPDS n $\left.{ }^{\circ} 5-592\right)$. In spite of been a commercially available product, the burden of the lab preparation of this sulfide is rewarding, since sonochemically prepared $\mathrm{PbS}$ powders are more uniform in size than those commercially available 9 . From the SEM image shown in Figure 2, it can be seen that the $\mathrm{PbS}$ consists of aggregated nanoparticles, whose size was found in the range of 50-100 nm. Since crystalline $\mathrm{PbS}$ powder was obtained we propose that the formation of this particle probably occurs in the interfacial region between the cavitation bubbles and the surround bulk solution. Though the temperature in the interfacial region is much lower than interior of the collapsing bubbles, it is still high enough to rupture chemical bonds and induce a variety of reactions and formation of crystalline products ${ }^{21,22,23}$.

\subsection{Radio-stabilizing action of PbS-NP in PMMA}

Gamma irradiated PMMA exhibited lower $M_{v}$ values then unirradiated PMMA and the degradation index (DI) was found to be 0.383 (see Table 1), which characterizes the main chain scission effect. This result agrees with literature reports on the effect of gamma radiation on PMMA matrix ${ }^{12,17}$. During PMMA irradiation, both electronic excitation and ionization processes produces excited atoms and ions which lead to the release of side groups like $\mathrm{CH}_{3} \mathrm{OOC} \cdot$ and main chain scissions ${ }^{12,14}$. It was reported that initial side-chain scissions generate macro radicals that further undergo main chain scissions ${ }^{12,19}$ and is the basic reason for the radiation-induced degradation of PMMA. In air, the macro radical undergoes chain oxidation process forming peroxyl radical subsequently it can abstract hydrogen from PMMA chains to form hydroperoxides. Then the hydroperoxides decomposes slowly, but steadily at room temperature to generate new oxidative products, inducing further degradation. In addition, it is believed that macro radical, peroxyl radical,

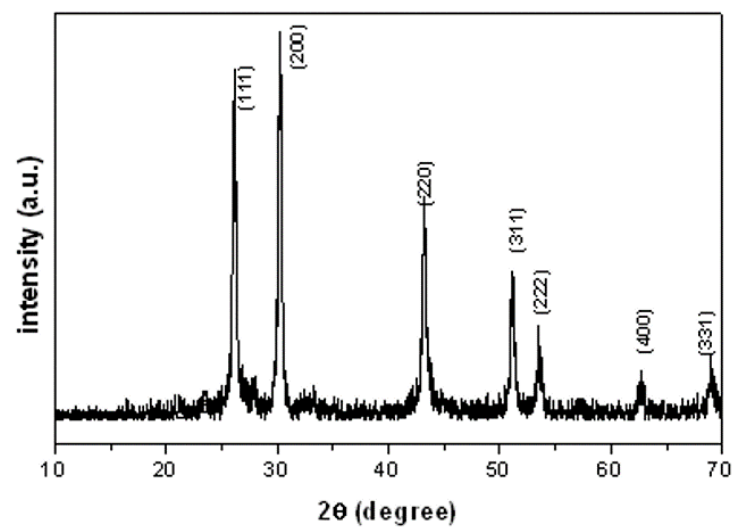

Figure 1. DRX pattern of the $\mathrm{PbS}$ powder.

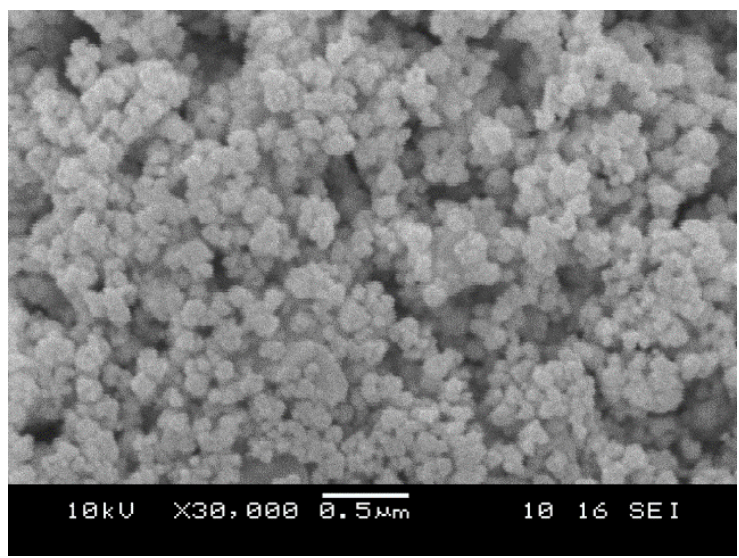

Figure 2. SEM image of PbS-NP.

Table 1. Viscosity results for PMMA films.

\begin{tabular}{ccc}
\hline $\begin{array}{c}\text { Concentration of } \\
\text { PbS (wt\%) }\end{array}$ & DI & Protection (\%) \\
\hline 0.00 & 0.383 & - \\
0.15 & 0.251 & 34 \\
0.30 & 0.000 & 100 \\
0.45 & 0.242 & 37 \\
0.60 & 0.291 & 24 \\
\hline
\end{tabular}


and hydroperoxides are the main substances responsible for changes in properties of gamma irradiated PMMA.

For radiation resistance study the Figure 3 shows $M_{v}$ for the PMMA-control and PMMA/PbS-NP before and after irradiation. The data revealed the decrease in the $\mathrm{M}_{\mathrm{v}}$ for both systems and it is expected, because, when already discussed, the main chain scission is the principal effect of the gamma radiation in the PMMA molecules. However the decrease in chain scissions occurred in PMMA/PbS-NP films, in special at $0.30 \mathrm{wt} \%$ of $\mathrm{PbS}$ concentration. In this concentration the $M_{v}$ value not undergoes changes after gamma irradiation. These data represent a decrease of $100 \%$ in scissions per original molecule of PMMA, evidencing an effective radiolytic stabilization of PMMA matrix owed to the presence of $\mathrm{PbS}-\mathrm{NP}$.

In addition, the concentration of PbS-NP in polymer matrix has an important role in the radiolytic stabilization. The Table 1 shown the DI calculated for all concentrations of $\mathrm{PbS}$ studied and a decrease of the PbS-NP stabilizing action was observed with the increase of nanoparticles concentration in the PMMA matrix. A probable explanation is a complex reactions can were catalyzed by nanoparticles, which can promotes PMMA degradation when it is present in higher concentrations ${ }^{24,25}$.

To the best of our knowledge, no information about the use of $\mathrm{PbS}-\mathrm{NP}$ in radiolytic stabilization of polymers has been published so far. Consequently, its mechanism of action has not been elucidated yet. However, some inferences of possible reactions under gamma irradiation may be done. Radical species formed during auto-oxidation on air-irradiated PMMA are peroxyl macroradicals. An effective inhibitor is required to interrupt this formation process. The efficiency of certain compounds in the stabilization of polymer molecules against radiation may be inferred by measuring the effect of these compounds on the radical population after irradiation, as well as on its rate of decay. A feasible mechanism of PMMA radio-stabilization could attribute radical scavenging characteristics to PbS-NP in the specific concentration. In this way, a suitable compound to assess such capability is DPPH, an artificial stable radical. Thus, the DPPH radical scavenging assay presents itself as a test of prediction of the antioxidant potential activity. The assay is based on the DPPH property of presenting a strong absorption at visible spectrum in $515 \mathrm{~nm}$, characterized by an intense violet

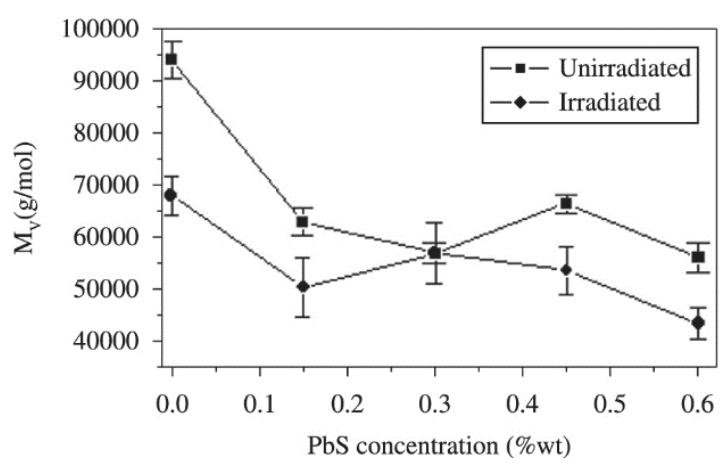

Figure 3. Viscosity molar mass $\left(\mathrm{M}_{\mathrm{v}}\right)$ of PMMA in function of $\mathrm{PbS}-\mathrm{NP}$ concentration. coloration, due to the presence of unpaired electrons. The absorbance obtained for DPPH solution in this study is $1.37 \pm 0.04$. When in the presence of substances capable of scavenging radicals, DPPH absorption is inhibited, leading to a stoichiometric discoloration in relation to the number of DPPH reduced molecules. The degree of discoloration is directly correlated with the radical scavenging activity of the evaluated substance ${ }^{26}$.

For test our radical scavenging hypothesis, we performed $\mathrm{DPPH}$ assay in PbS-NP solutions. Our results revealed that $\mathrm{PbS}-\mathrm{NP}$, in the amount of $0.0054 \mathrm{~g}$ (equivalent to concentration of $0.3 \mathrm{wt} \%$ in PMMA matrix) have radical scavenging action, with $64 \%$ of radical capture, thus explaining their radiolytic stabilizing action on PMMA matrix. Probably, DPPH radical abstracts one electron from the sulfur atom and oxidizes it to the short-lived radical R, which reacts with DPPH in the same way shown in Scheme 1, yielding the final derivative of $\mathrm{DPPH}^{27,28}$. This result is compared with BHT (same amount, i.e., $0.0054 \mathrm{~g}$ ), which have around $99 \%$ of radical capture. Table 2 shows the results obtained by use of PbS-NP as a radical scavenger on DPPH solution.

The results obtained by DPPH tests are satisfactory considering the small amount of PbS-NP added to the system. Thus, the nanoparticles used in this study may be considered as an additive with stabilizing action on PMMA molecules. We assumed the radical scavenging action to be the main role of PbS-NP on the films of PMMA, but further work is required to a better understanding of the overall radiostabilizing process involving PbS-NP in PMMA matrix.

\subsection{PMMA/PbS-NP films characterization}

In this study we choice a film casting in which PbS-NP were dissolved in a PMMA-MEK solution forming films upon evaporation of the solvent. The film casting method is
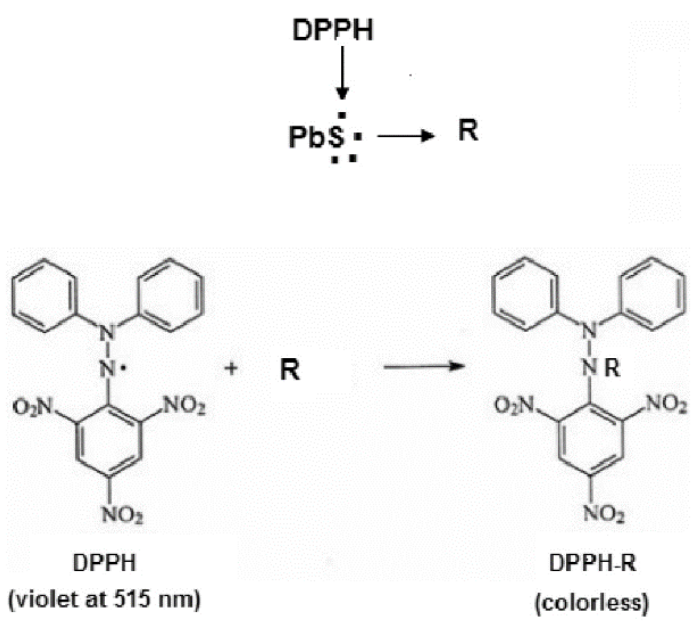

Scheme 1. Capture mechanism of DPPH radical.

Table 2. Radical DPPH scavenging capacity (SC) of PbS-NP.

\begin{tabular}{lcc}
\hline \multicolumn{1}{c}{ System } & Absorbance & SC (\%) \\
\hline $\mathrm{DPPH}$ & $1.37 \pm 0.04$ & - \\
$\mathrm{DPPH}+\mathrm{PbS}-\mathrm{NP}$ & $0.73 \pm 0.02$ & 64 \\
$\mathrm{DPPH}+\mathrm{BHT}$ & $0.38 \pm 0.03$ & 99 \\
\hline
\end{tabular}


widely used due to its simplicity and results in the formation of homogeneous films. Figure 4 shows SEM images of PMMA/ $\mathrm{PbS}-\mathrm{NP}$ (unirradiated) at $0.3 \mathrm{wt} \%$ concentration exhibiting a nanoparticles distribution. The surface morphology of the PMMA/PbS-NP shows some aggregates nanoparticles or chunks randomly distributed in the film. The results indicate that the PbS-NP tended to form aggregates that were dispersed into the PMMA matrix. PbS particle size allows a more homogeneous distribution (see Figure 2) of a PMMA/PbS-NP material and leads to a drastic increase of the polymer-nanoparticle interfacial area due to the high specific surface area of nanoparticles. This may induce aggregation of the nanoparticles to energetically stabilize PMMA/PbS-NP system, thus lowering the homogeneity of particle distribution. In addition, the SEM image of irradiated film presented similar behavior than Figure 4 and not are shown in this paper.

The distribution of $\mathrm{PbS}(0.3 \mathrm{wt} \%)$ in the PMMA matrix is an important factor for PMMA/PbS-NP application, however the interactions between the nanoparticles and polymer molecules play an important role for its properties. The FT-IR spectra of PMMA-control and PMMA/PbS-NP are shown in Figure 5 and show the main expected bands characterizing the vibration spectrum of PMMA [29]. In the

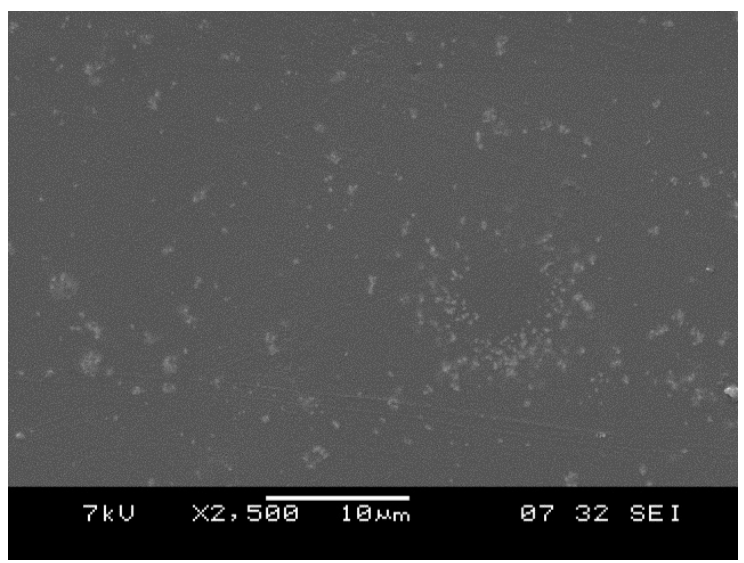

Figure 4. SEM image of PMMA/PbS-NP films.

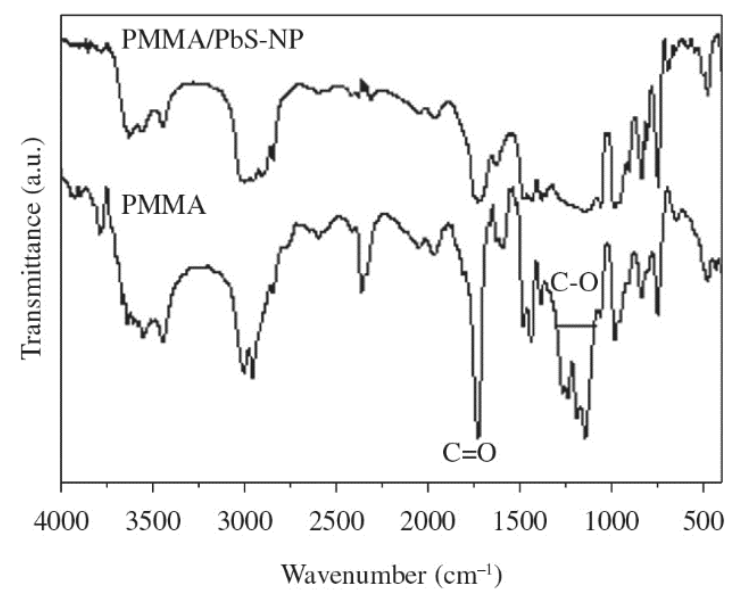

Figure 5. FT-IR spectra of PMMA-control and PMMA/PbS-NP films.
PMMA spectrum the two bands at $3000 \mathrm{~cm}^{-1}$ and $2960 \mathrm{~cm}^{-1}$ can be assigned to the $\mathrm{C}-\mathrm{H}$ bond stretching vibrations of the $-\mathrm{CH}_{3}$ and $-\mathrm{CH}_{2}-$ groups, respectively. The band at $1731 \mathrm{~cm}^{-1}$ shows the presence of the acrylate carboxyl group. The two bands at $1387 \mathrm{~cm}^{-1}$ and $755 \mathrm{~cm}^{-1}$ can be attributed to the $\alpha$-methyl group vibrations. The band at $1443 \mathrm{~cm}^{-1}$ can be attributed to the bending vibration of the $\mathrm{C}-\mathrm{H}$ bonds of the $-\mathrm{CH}_{3}$ group. There is a distinct absorption band from $1145 \mathrm{~cm}^{-1}$ to $1271 \mathrm{~cm}^{-1}$, which can be attributed to the $\mathrm{C}-\mathrm{O}-\mathrm{C}$ stretching vibration. The band at $987 \mathrm{~cm}^{-1}$ is the characteristic absorption vibration of PMMA, together with the bands at $1062 \mathrm{~cm}^{-1}$ and $843 \mathrm{~cm}^{-1}$.

Interaction between polymer and nanoparticles may shift the polymer cage peak frequencies. FT-IR technique is would be sensitive both in situations where interactions has occurred in crystalline or amorphous phase ${ }^{29}$. In the PMMA/ $\mathrm{PbS}-\mathrm{NP}$ spectrum the peaks of pure PMMA at $1731 \mathrm{~cm}^{-1}$ are shifted to $1726 \mathrm{~cm}^{-1}$ and changes such as broadening of the $\mathrm{C}=\mathrm{O}$ and $\mathrm{C}-\mathrm{O}-\mathrm{C}$ peaks and the decrease of their intensities can also be observed. These results mean that the predicted interactions between PMMA and PbS-NP have indeed taken place. Thus, the adsorption of PbS-NP on PMMA molecules occurs near to carbonyl group ${ }^{30,31}$, probably between the $\mathrm{Pb}$ atom in PbS-NP and the oxygen atom in PMMA molecule.

Ours conclusion about interaction of PMMA and PbS-NP may be reinforced by viscosity analysis. There is a direct relationship between $\mathrm{M}_{\mathrm{v}}$ value and the intrinsic viscosity (see Equation 2). For example, the increase in $M_{v}$ value means an increase in intrinsic viscosity ${ }^{14,16,32}$. The Figure 3 showed a decrease of $\mathrm{M}_{\mathrm{v}}$ of PMMA/PbS-NP when compared to $\mathrm{M}_{\mathrm{v}}$ of PMMA-control for both irradiated and unirradiated films. The decrease in intrinsic viscosity showed in unirradiated films indicates the contraction of the PMMA coil in solution, most probably due interactions between $\mathrm{Pb}$ atom and two or more carbonyl group, which have binding site and have dipole-dipole interaction and cause coiling of molecule. The shrunken compact coil of PMMA and PbS-NP can easily pass through the capillary of viscometer compared to polymer in absent of nanoparticles and thus a decrease in viscosity occurs. Thus, the results about interactions between PbS-NP and PMMA discussed from viscosity analyses agree with FT-IR results.

\subsection{Mechanical and optical properties}

PMMA systems were gamma irradiated and changes were investigated in order to analyze the irradiation and PbS-NP (0.3 wt $\%$ ) effects on mechanical properties of polymer systems. The results of elongation at break $(\mathrm{Eb})$ and Young's modulus (Ym) mechanical tests for PMMA and PMMA/PbS-NP are summarized in Table 3.

Table 3. Effects of PbS-NP and gamma irradiation in mechanical properties of PMMA.

\begin{tabular}{lccc}
\hline Systems & $\begin{array}{c}\text { Dose } \\
(\mathbf{k G y})\end{array}$ & $\begin{array}{c}\text { Ym } \\
(\mathbf{M p a})\end{array}$ & $\begin{array}{c}\text { Ea } \\
(\mathbf{\%})\end{array}$ \\
\hline PMMA & 0 & $790.10 \pm 92.24$ & $8.50 \pm 2.33$ \\
& 25 & $595.83 \pm 61.08$ & $9.00 \pm 1.66$ \\
PMMA/Pb & 0 & $454.30 \pm 13.22$ & $9.30 \pm 1.36$ \\
& 25 & $420.53 \pm 13.54$ & $9.15 \pm 1.41$ \\
\hline
\end{tabular}


The results showed decrease in Young's modulus values of PMMA/PbS-NP as compared to the control PMMA for unirradiated systems. This result means a decrease in rigidity of the PMMA and consequently explains the increase of $6 \%$ on $\mathrm{Eb}$ value of $\mathrm{PMMA} / \mathrm{Pb}-\mathrm{NP}$. Thus, the $\mathrm{PbS}$ nanoparticles act as a plasticizer in the PMMA molecule. Since interactions between PMMA matrix and PbS-NP were found in the FT-IR and viscosity analysis, these interactions can cause increase of mobility of the polymers molecules giving them the plasticizing effect ${ }^{30,33}$.

The changes in properties of irradiated samples may be attributed to the radiation-induced oxidative degradation of the PMMA molecules. For the films of PMMA irradiated was found a decrease of $42.5 \%$ in Ym value with consequent increase in $\mathrm{Eb}$ value. The chain scission effect obtained by gamma irradiation (Figure 3) provokes the decrease of average length of PMMA molecule. The density of entanglements points decreases leading to a decrease of the Ym value as consequence of PMMA radiolytic degradation. The lower molecular weight also makes fibrils less stable and therefore favors brittle fracture ${ }^{33}$. On the other hand, no significant changes of $\mathrm{Ym}$ and $\mathrm{Eb}$ values were found for irradiated PMMA/PbS-NP. These results are in accordance with the radio-stabilizing action of PbS-NP evidenced by viscosity analysis.

On the other hand, the optical property of PMMA also undergoes changes with the PbS-NP presence. Optical applications of polymers are often limited due to the relatively narrow range of the refractive index (RI) for high RI polymer nanocomposites, where the RI found is above 1.5. The RI values of most commercial polymers are typically around 1.3, while inorganic materials as $\mathrm{PbS}$ can possess $\mathrm{RI}$ above $3^{11}$. The RI found for PMMA-control and PMMA/PbS-NP films are shown in Figure 6. The RI of PMMA-control, for example, is found 1.4911 while 1.5045 is the RI for PMMA/ $\mathrm{PbS}-\mathrm{NP}$ at $0.6 \mathrm{wt} \%$ concentration, for example. Thus, the introduction of PbS-NP nanoparticles into a PMMA matrix can result in polymeric nanocomposites with higher RI. The irradiation provokes a slight decrease in the RI of PMMAcontrol probably by presence of trapped radicals formed from polymer degradation. These radicals are color centers that absorb light in the ultraviolet and visible range ${ }^{12,13,14}$. Some of the radicals are quenched after irradiation by annealing

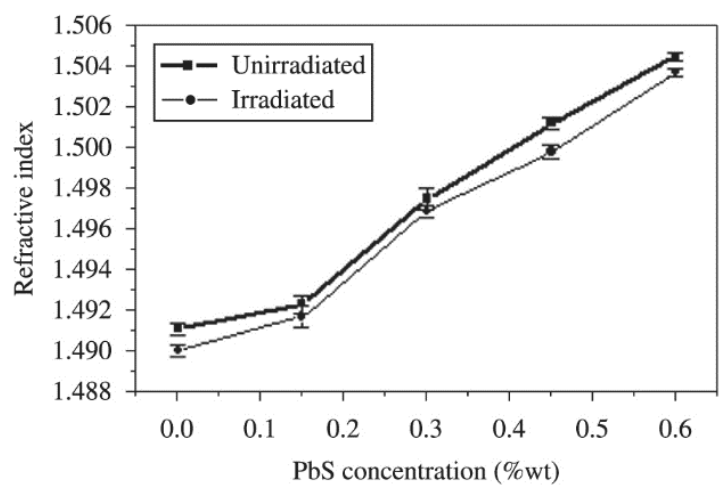

Figure 6. Refractive index of PMMA in function of PbS-NP concentration. reactions with oxygen that diffuses into the matrix. However this effect is minimized by PbS-NP presence and its radical scavenger action may be an acceptable explication.

\subsection{Thermogravimetric analysis of PMMA/PbS- NP films}

Ours previously study ${ }^{15}$ showed that the PMMA in study contains a double bond in the end group, which is formed through radical polymerization of $\mathrm{PMMA}^{34,35}$. In general, under inert atmosphere, thermal degradation of double bond-terminated PMMA (PMMA=) prepared by radical polymerization exhibits four main weight losses events, corresponding to four degradation stages ${ }^{34,35,36}$. The first stage corresponds to the degradation initiated by radical transfer to the unsaturated chain end; the second and third stages result from the homolytic scission of the chain due to headto-head bonds and degradation initiated by radical transfer to the unsaturated chain end; the fourth stage corresponds to random scissions ${ }^{15}$.

TGA thermograms of PMMA and PMMA/PbS-NP $(0.3 \mathrm{wt} \%)$ are shown in Figure $7 \mathrm{a}$ for unirradiated systems. TG curves obtained for PMMA and PMMA/PbS-NP samples were found in a single step of thermal decomposition, i.e, the addition of PbS-NP does not cause changes in the polymer thermal behavior. Figure 7a shows a great thermal stabilization of PMMA/PbS-NP system because the incorporation of the PbS-NP shifts TGA curves to higher temperatures. We found an increase of $46^{\circ} \mathrm{C}$ and $49^{\circ} \mathrm{C}$ in the $\mathrm{T}_{50}$ and $\mathrm{T}_{\mathrm{m}}$, respectively of PMMA/PbS-NP films (see Table 4).

FT-IR (Figure 5) and viscosity results showed that some interactions between PMMA and PbS-NP might exist. These interactions might exist at specific discrete locations

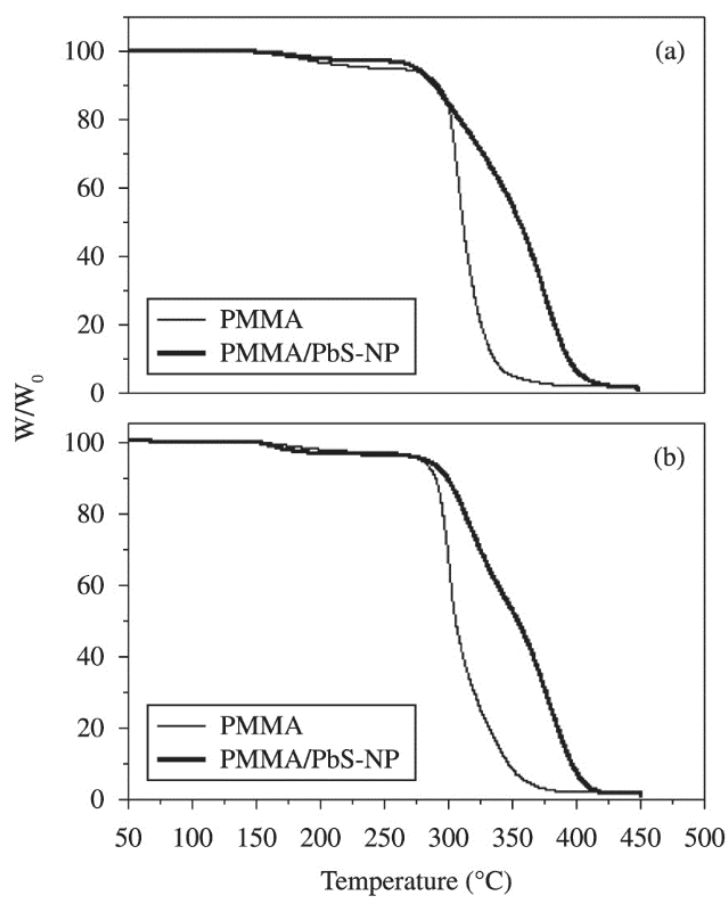

Figure 7. TGA curve of PMMA-control and PMMA/PbS-NP for a) unirradiated and b) irradiated films. 
Table 4. TGA results for PMMA and PMMA/PbS-NP films.

\begin{tabular}{cccc}
\hline Systems & $\begin{array}{c}\text { Dose } \\
(\mathbf{k G y})\end{array}$ & $\begin{array}{c}\mathbf{T}_{\mathbf{5 0}} \\
\left({ }^{\circ} \mathbf{C}\right)\end{array}$ & $\begin{array}{c}\mathbf{T}_{\mathbf{m}} \\
\left({ }^{\circ} \mathbf{C}\right)\end{array}$ \\
\hline PMMA-control & 0 & 311 & 330 \\
& 25 & 305 & 325 \\
PMMA/Pb-NP & 0 & 357 & 379 \\
& 25 & 352 & 370 \\
\hline
\end{tabular}

where the carbonyl group of PMMA molecule interacts with nanoparticles. Thus the interactions increase the mobility of polymers molecules (discussed for mechanical properties), but change the mobility of the ester group. This group favors the radical transfer to the unsaturated chain and facilitates the scission of the PMMA=. The decrease in the mobility of ester group due it interaction with $\mathrm{PbS}-\mathrm{NP}$ may explained the stabilizer action of PbS-NP.

TGA thermograms of irradiated PMMA-control and PMMA/PbS-NP are shown in Figure 7b. Films irradiated also showed a single step of thermal decomposition and exhibited smaller $\mathrm{T}_{50}$ and $\mathrm{T}_{\mathrm{m}}$ temperatures than unirradiated samples (see Table 4), probably owed to radiolytic degradation of the films. When PMMA and PMMA/PbS-NP films are gamma irradiated, they undergo chains scissions. In this way, radiolysis products may play an important role in the acceleration of thermal degradation of PMMA molecules. However, we also found an increase of $47^{\circ} \mathrm{C}$ and $45^{\circ} \mathrm{C}$ in the $\mathrm{T}_{50}$ and $\mathrm{T}_{\mathrm{m}}$, respectively of PMMA/PbS-NP gamma irradiated. Thus, the thermal stabilization action of PbS-NP is also significant in irradiated systems.

\section{References}

1. Brus LEA. Quantum crystallites and nonlinear optics. Applied Physics. 1991; 53(6):465-474. http://dx.doi.org/10.1007/ BF00331535.

2. Kruis FE, Fissan $H$ and Rellinghaus B. Sintering and evaporation characteristics of gas-phase synthesis of size-selected $\mathrm{PbS}$ nanoparticles. Materials Science and Engineering B. 2000; 6970:329-334. http://dx.doi.org/10.1016/S0921-5107(99)00298-6.

3. Fujimoto M and Sato YPT. $x$ phase diagram of the lead telluride system. Japanese Journal of Applied Physics. 1966; 5(2):128133. http://dx.doi.org/10.1143/JJAP.5.128.

4. Xie Y, Qiao Z, Chen M, Zhu Y and Qian Y. y-irradiation route to nanocrystalline lead selenide. Chemistry Letters. 1999; 9:875-876. http://dx.doi.org/10.1246/cl.1999.875.

5. Eastoe J and Cox RA. Formation of PbS nanoclusters using reversed micelles of lead and sodium aerosol-OT. Colloids and Surfaces. A, Physicochemical and Engineering Aspects. 1995; 101(1):63-76. http://dx.doi.org/10.1016/0927-7757(95)03177-F.

6. Zhao XK, Xu SQ and Fendler JH. Semiconductor particles formed at monolayer surfaces. Langmuir. 1991; 7(3):520-524. http://dx.doi.org/10.1021/la00051a018.

7. Henshaw G, Parkin IPG and Shaw G. Shaw Elemental, liquid ammonia facilitated routes to zinc, cadmium, mercury copper, silver and lead telluride. Journal of Materials Science Letters. 1996; 15(20):1741-1742. http://dx.doi.org/10.1007/BF00275327.

8. Kane RS, Cohen RE and Silbey R. Synthesis of PbS nanoclusters within copolymer nanoreactors. Chemistry of Materials. 1996; 8(8):1919-1924. http://dx.doi.org/10.1021/cm960072i.
In general, a comparison between irradiated and unirradiated systems showed that irradiation caused decrease in $\mathrm{T}_{50}$ of $2 \%$ for PMMA-control and only around $1 \%$ for PMMA/PbS-NP. Such findings corroborate the radiolytic stabilization action PbS-NP in the PMMA matrix.

\section{Conclusion}

The PbS-NP were synthesized through sonochemical method as nanocrystalline powder. The dispersion of these nanoparticles in PMMA matrix at low concentration (0.3 wt \%) resulted $100 \%$ stabilization of polymer degradation. DPPH tests evidenced that PbS-NP act as radical scavengers, suggesting this mechanism as the most probable way of its stabilizing effect. Intrinsic viscosity and FT-IR spectra of PMMA films containing PbS-NP indicated interactions between these two components specifically near of carbonyl group of PMMA. Such interactions might favor the improvement of mechanical and thermal properties of $\mathrm{PMMA} / \mathrm{PbS}-\mathrm{NP}$ system. In addition, the introduction of PbS-NP nanoparticles into a PMMA matrix can result in polymeric nanocomposites with higher refractive index. These results are opening pathways for engineering flexible polymer-inorganic mixture that exhibit advantageous optical, thermal and mechanical properties with radiation resistance.

\section{Acknowledgements}

We would like to thanks CNPq-Brazil for financial support and the Resarbrás-Brazil for PMMA samples.

9. Suslick KS. Sonochemistry. Science. 1990; 247(4949):14391445. http://dx.doi.org/10.1126/science.247.4949.1439. PMid:17791211

10. Suslick KS, Choe SB, Cichowlas AA and Grinstall MW. Sonochemical synthesis of amorphous iron. Nature. 1991; 353(6343):414-416. http://dx.doi.org/10.1038/353414a0.

11. Zimmermann L, Weibel M, Caseri W and Suter UW. Polymer nanocomposites with "ultralow" refractive index. Journal of Materials Research. 1993; 8:1742-1748. http://dx.doi. org/10.1557/JMR.1993.1742.

12. Suarez JCM, Mano EB, Monteiro EEC and Tavares MIB. Influence of $\gamma$-irradiation on poly(methyl mthacrylate). Journal of Applied Polymer Science. 2002; 85(4):886-895. http://dx.doi. org/10.1002/app.10706.

13. Ishigaki I and Yoshi F. Radiation effects on polymer materials in radiation sterilization medical supplies. Radiation Physics and Chemistry. 1992; 39:527-533.

14. Dole M. The radiation chemistry of macromolecules. New York: Academic Press; 1973. v. 2.

15. Aquino KAS and Araujo ES. Effects of a Hindered Amine Stabilizer (HAS) on radiolytic and thermal stability of Poly(methyl methacrylate). Journal of Applied Polymer Science. 2008; 110(1):401-407. http://dx.doi.org/10.1002/app.28465.

16. Schnabel W. Polymer degradation-principles and practical applications. New York: Macmillan Publishing; 1981.

17. Miller KJ, Hellman JH and Moore JA. Conformation of poly(methyl methacrylate) and its degraded forms upon radiation. Macromolecules. 1993; 26(18):4945-4952. http:// dx.doi.org/10.1021/ma00070a031. 
18. Cragg LH and Bigelow CC. The viscosity slope constant K-ternary systems: Polymer-polymer-solvent. Journal of Polymer Science. Polymer Physics Edition. 1955; 16:177-191.

19. Guillet J. Polymer photophysics and photochemistry. New York: Cambridge University Press; 1985.

20. Brandrup J and Immergut EH. Polymer handbook. New York: John Wiley Sons; 1989.

21. McNamara III, Didenko WBY and Suslick KS. Sonoluminescence temperatures during multibubble cavitation. Nature. 1999; 401(6751):772-775.

22. Jeevanandam P, Koltypin Y, Gedanken A and Mastai Y. Synthesis of $\alpha$ cobalt (II) hydroxide using ultrasound radiation. Journal of Materials Chemistry. 2000; 10(2):511-514. http://dx.doi. org/10.1039/a908065a.

23. Gautam U and Mukherjee K. Preparation of Al-Sb semiconductor by swift heavy ion irradiation. B. Bulletin of Materials Science. 2006; 29:1-5. http://dx.doi.org/10.1007/BF02709345.

24. Lee EH, Rao GR and Mansur LK. LET effects on cross-linking and scission mechanism of PMMA during irradiation. Radiation Physics and Chemistry. 1999; 55(3):293-305. http://dx.doi. org/10.1016/S0969-806X(99)00184-X.

25. La Mantia FP, Dintcheva NT, Malatesta V and Pagani F. Improvement of photo-stability of LLDPE-based nanocomposites. Polymer Degradation \& Stability. 2006; 91(12):3208-3213. http://dx.doi.org/10.1016/j.polymdegradstab.2006.07.014.

26. Mathiesen L, Malterud KE and Sund RB. Hydrogen bond formation as basis for radical scavenging activity: a structureactivity study of C-methylated dihydrochalcones from Myrica gale and structurally related acetophenones. Free Radical Biology \& Medicine. 1997; 22(1-2):307-311. http://dx.doi. org/10.1016/S0891-5849(96)00277-8. PMid:8958155

27. Ionita P, Constantinescu T, Caldararu H, Luca C, Caproiu MT, Dumitrascu F, et al. Reaction between the DPPH free radical and potassium cyanide in the presence of crown ether 18-C-6: a correction. Revue Roumaine de Chimmie. 1999; 44:393-396.

28. Ionita $\mathrm{P}$, Spafiu F, Constantinescu $\mathrm{T}$ and Caldararu $\mathrm{H}$. The reaction of 2,2-diphenyl-1-picrylhydrazyl stable free radical with sodium tetra-phenylborate in the presence of $18-\mathrm{C}-6$ crown ether. Revue Roumaine de Chimmie. 1999; 44:497-500.

29. Ramesh S, Leen KH, Kumutha K and Arof AK. FTIR studies of PVC/PMMA blend based polymer electrolytes. Spectrochimica Acta Part A. 2007; 66(4-5):1237-1242. http://dx.doi.org/10.1016/j. saa.2006.06.012. PMid:16919998

30. Tadd E, Zeno A, Zubris M, Dan N and Tannenbaum R. Adsorption and polymer film formation metal nanoclusters. Macromolecules. 2003;36(17):6497-6502. http://dx.doi.org/10.1021/ma034207z.

31. Tannenbaum R, Zubris M, Goldberg EP, Reich S and Dan N. Polymer-directed nanocluster synthesis: control of particle size and morphology. Macromolecules. 2005; 38(10):4254-4259. http://dx.doi.org/10.1021/ma048317x.

32. Khan MS, Gul K and Rehman NU. Interaction of polyvynilpyrrolidone with metal choride aqueous solutions. Chinese Journal of Polymer Science. 2004; 22:581-584.

33. Lipatov YS. Polymer reinforcement. Canada: ChemTec Publishing; 1995.

34. Manring LE. Thermal degradation of poly(methyl methacrylate).2.Vinyl terminated polymer. Macromolecules. 1989; 22(6):2673-2677. http://dx.doi.org/10.1021/ma00196a024.

35. Manring LE, Sogah DY and Cohen GM. Thermal degradation of poly(methyl methacrylate): 3. Polymer with head-to-head linkages. Macromolecules. 1989; 22(12):4652-4654. http:// dx.doi.org/10.1021/ma00202a048.

36. Schnabel W. Polymer degradation: principles and practical applications. Munich: Carl Hanser Verlag; 1982. 